\title{
Design and characterization of the FAST chip: a front-end for 4D tracking systems based on Ultra-Fast Silicon Detectors aiming at 30 ps time resolution
}

\author{
E.J. Olave ${ }^{a, *}$, F. Fausti ${ }^{\text {a,d }}$, N. Cartiglia ${ }^{a}$, R. Arcidiacono ${ }^{a, b}$, H.F.-W. Sadrozinski ${ }^{c}$, A. Seiden $^{c}$ \\ a INFN sezione di Torino, via P. Giuria 1, 10125, Torino, ITA, Italy \\ ${ }^{\mathrm{b}}$ Universita del Piemonte Orientale, Rettorato, via Duomo, 6, 13100 Vercelli, ITA, Italy \\ ' Santa Cruz Institute for Particle Physics, University of California (SCIPP), Natural Sciences 2, 1156 High Street, Santa Cruz, CA 95064, Italy \\ d DE.TEC.TOR. Devices \& Technologies Torino S.r.l., Lungo Dora Voghera, 36/A, 10153 Torino, Italy
}

\section{A R T I C L E I N F O}

\section{Keywords:}

Analog readout electronics

Timing detectors

Silicon sensors

\begin{abstract}
A B S T R A C T
Detectors able to measure the time of flight with very high accuracy ( $\sim 10$ ps RMS) are becoming fundamental in the design of new High Energy Physics experiments, where accurate time measurements will be used to mitigate pileup effects. The development of such detectors has spurred intense R\&D in both silicon sensors and the associated readout electronics, aiming at obtaining silicon-based detectors with a time resolution in the few tens-of-picosecond range. This work presents FAST, a family of three different 20 channel amplifier-comparator chips, tailored to the readout of Ultra Fast Silicon Detectors. These ASICs have been designed optimizing the sensor-readout interplay with the aim of reaching the smallest possible jitter term. The three chips of the FAST family differ in the architecture of the front-end while sharing the channel back-end, consisting of a leading-edge discriminator and a LVDS driver. The goal of these front-ends is to achieve a time resolution of about 30 ps RMS while coupled to a sensor with a few pico-Farad capacitance, keeping the power budget of the single channel below $1.3 \mathrm{~mW}$. This paper reports the description of the FAST design architecture and summarizes the results on the initial characterization of one chip of the FAST family, in a stand-alone test structure and when coupled to a UFSD.
\end{abstract}

\section{Introduction}

The future High Energy Physics (HEP) experiments are seeking to achieve higher and higher luminosity, to maximize the potential for new discoveries. As a consequence, the rate of events is going to be so high that multiple concurrent collisions will reduce the final reconstruction accuracy. The problem of overlapping events, known as pileup, requires dedicated mitigation techniques. During the Large Hadron Collider (LHC) Run I and during the beginning of Run II, techniques such as the area-mean [1] have been very efficient in achieving pileup mitigation. However, in the future High Luminosity-LHC (HL-LHC) the average number of pileup interactions will increase from 60 to 200, and new techniques must be adopted to cope with overlapping events. The introduction of time measurements with a few tens of pico-second accuracy is a new tool that experiments are going to exploit. Examples of timing detectors planned in future HEP experiments are the High Granularity Timing Detector (HGTD) [2] and the MIP Timing Detector (MTD) [3]. Timing detectors with such demanding resolution need the development of dedicated sensors and electronics. Good options for timing sensors to reach 30 ps RMS time resolution are the so-called Ultra Fast Silicon Detectors (UFSD) [4,5], that are time-optimized
Low Gain Avalanche Diodes. The main requirement for the readout electronics is that it degrades the timing properties of the sensor as little as possible. This request leads to the design of a front-end that minimize the jitter due to the electronics contributing to the overall time resolution. Past readout chips designed for timing applications, for example those reported in [6,7], proved that a time resolution of 4050 ps RMS can be reached using $\sim 30 \mathrm{~mW}$ per channel. However, large detector systems need to employ much lower power budget while still reaching even lower time resolutions: the combination of low power consumption and good time resolution presents a formidable challenge. A review of a few projects exploring this challenge is presented in [8]. To increase the difficulty of this task, the capacitance of the sensors employed both in HGTD and MTD is quite large, 3-4 pF.

This paper presents the design, simulation and laboratory tests results of a family of new timing ASICs called FAST, developed to study front-end architectures with a power consumption of $1.3 \mathrm{~mW}$ and suitable to reach pico-second time resolution when coupled to $55 \mu \mathrm{m}$ thick UFSD with $2-6 \mathrm{pF}$ of capacitance. The next section reports an overview of the approach adopted during the design of amplifiers for timing applications. In Section 3 the front-end architectures used in the

\footnotetext{
* Corresponding author.

E-mail address: olave@to.infn.it (E.J. Olave).
} 


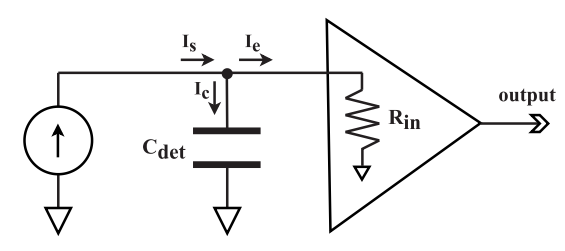

Fig. 1. Matching scheme between silicon sensor and front-end.

FAST family are described while the experimental results of one design of the FAST family, tested both stand-alone and combined with UFSD, are discussed in the last section.

\section{Amplifiers for timing}

The jitter is often expressed in literature as the noise-to-slope ratio and it can be minimized by a front-end able to generate an output with low noise $\sigma_{v}$ and high slope $\partial V / \partial t$. High slopes are obtained with a high amplitude and a short front-end peaking time $\left(\mathrm{t}_{p, f e}\right)$ of the analog signal [9]. Important quantities that enter in the jitter minimization are the amplifier bandwidth BW, the sensor-front-end impedance matching, and the amplifier gain. The bandwidth affects both noise $(\propto \sqrt{B W})$ and slope ( $\propto \mathrm{BW})$ and, ideally, the higher BW the lower the jitter. However, the intrinsic time response $\tau_{s}$ of UFSD sensors sets the upper limit to the maximum reachable slope that the analog output can exhibit. As a consequence, the bandwidth should be chosen to be the minimum value that retains the intrinsic sensor speed while keeping the noise low. The bandwidth defines the signal shaping of the front-end and its optimum value for timing is obtained when $t_{p, f e}$ equals the sensor peaking time $\mathrm{t}_{p, s}$, which is the time needed by the signal induced by the charge collected into the sensor, to reach its maximum [9].

The sensor-front-end matching is a key ingredient for a successful front-end design. The input time constant of the amplifier, defined as $\tau_{i n}$ $=\mathrm{C}_{d e t} \cdot R_{i n}$, must be chosen according to $\tau_{s}$. Fig. 1 shows the important contributions in the matching. The sensor area and thickness affect the sensor capacitance $\mathrm{C}_{\text {det }}$ whereas the input impedance $\mathrm{R}_{\text {in }}$ depends on the front-end. With good matching, most of the current pulse generated by the sensor enters into the amplifier which operates in current mode, defined as $\mathrm{I}_{s}=\mathrm{I}_{e}$. This condition is obtained when $\tau_{\text {in }}<\tau_{s}[10]$. Considering the typical UFSD value of $\tau_{s}$ of $\sim 400$ ps (defined by the sensor collection time) for a $55 \mu \mathrm{m}$ thick sensor with capacitance of $\mathrm{C}_{d e t}$ $=4 \mathrm{pF}$, the front-end should be designed with $\mathrm{R}_{\text {in }}$ lower than $100 \Omega$. In case of a much larger sensor capacitance, the condition $\tau_{i n}<\tau_{s}$ is difficult to achieve because it will require too small values of $\mathrm{R}_{i n}$. In cases where $\mathrm{C}_{d e t} \cdot \boldsymbol{R}_{i n} \ll \tau_{s}$, the current $\mathrm{I}_{s}$ is entirely integrated on $\mathrm{C}_{d e t}$, generating a voltage pulse at the input of the amplifier. In this conditions, the maximum signal slope is given by the ratio between the maximum current $\mathrm{I}_{\max }$ generated by the sensor and $\mathrm{C}_{d e t}$. Taking as reference a $4 \mathrm{pF}, 55 \mu \mathrm{m}$ thick UFSD coupled to a front-end having $\mathrm{R}_{i n}$ $\gg 100 \Omega$, the most probable slope for an impinging MIP (Minimum Ionizing Particle) is about $2.75 \mathrm{mV} / \mathrm{ns}$.

Generally, current mode amplifiers are the best choice for preserving both the rising and falling edges of the fast sensor pulse. However, this is feasible only with small values of $\mathrm{C}_{d e t}$. Depending on the impedance matching, the peaking time $t_{p, s}$ of the pulse (current or voltage) sent to the amplifier from a $55 \mu \mathrm{m}$ thick UFSD ranges between 0.6 and $1.2 \mathrm{~ns}$. If these signals are amplified by a timing-optimized front-end, the expected peaking time, given by Formula (1), will range between 0.85 and $1.7 \mathrm{~ns}$.

$t_{p}=\sqrt{t_{p, s}^{2}+t_{p, e}^{2}} \stackrel{t_{p, s}=t_{p, e}}{\longrightarrow} \sqrt{2} \cdot t_{p, s}$

Fig. 2 shows a simulation of timing optimization performed by using the model of Fig. 1 with a $4 \mathrm{pF}$ sensor capacitance, the typical signals generated by $55 \mu \mathrm{m}$ thick UFSD of moderate gain of about 15 and a

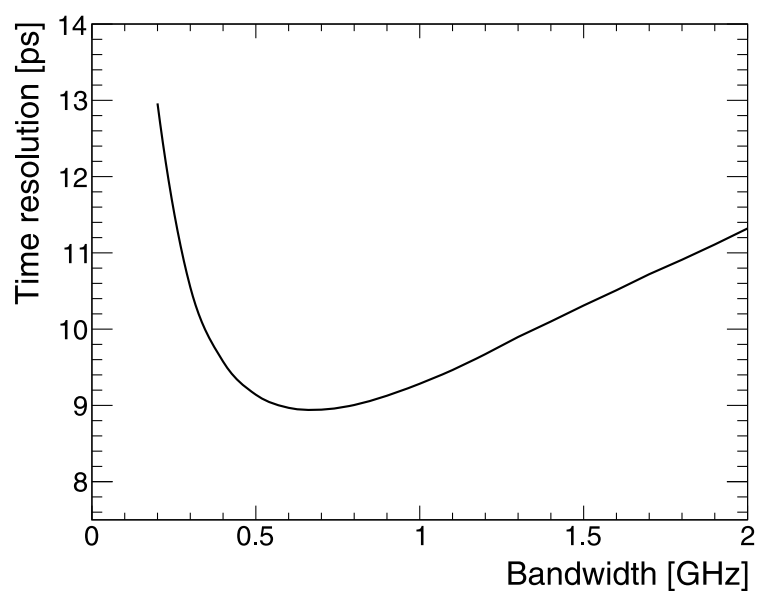

Fig. 2. Time resolution simulation based on the model of Fig. 1 where $C_{d e t}$ is $4 \mathrm{pF}$ and $\mathrm{R}_{i n}$ is $100 \Omega$. The total input charge is $8 \mathrm{fC}$.

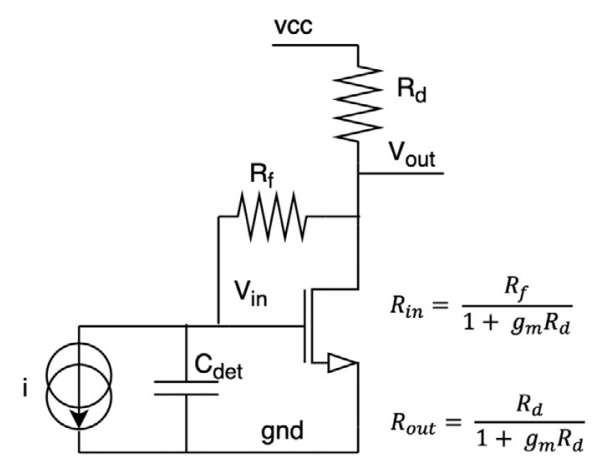

Fig. 3. Basic TIA scheme with the expressions that define $\mathrm{R}_{\text {in }}$ and $\mathrm{R}_{\text {out }}$.

two pole amplifier with an $\mathrm{R}_{\text {in }}$ equal to $100 \Omega$. The optimum bandwidth corresponds to values in the range of $400-800 \mathrm{MHz}$.

The final important term to consider in the design optimization is the amplified signal amplitude that should be maximized. In case of trans-impedance amplifiers (TIA), this can be obtained by using a large feedback resistance $\mathrm{R}_{f}$. However, since $\mathrm{R}_{\text {in }}$ increases with $\mathrm{R}_{f}$ as shown in Fig. 3, having a too large $\mathrm{R}_{f}$ degrades the sensor-front-end matching. For amplifiers with open loop gain of $40 \mathrm{~dB}$, a good tradeoff is achieved using $R_{f} \sim 5-20 \mathrm{k} \Omega$. In this case, $R_{i n}$ can be reduced by maximizing the input transistor transconductance $g_{m}$. For a constant power consumption, this can be ensured by increasing its width up to enter in the weak inversion region. Special care should be used during the front-end layout, using techniques to minimize undesired parasitics.

\section{The front-end architecture of FAST}

The three flavors of the FAST (Fast Amplifiers for Silicon detectors for Timing) family explore different front-end designs, each suitable to reach pico-second time resolutions. The flavors are labeled Regular, EVO1 and EVO2 and have been designed by using two architectures with different bandwidths. Regular has a bandwidth of $100 \mathrm{MHz}$ and aims at minimizing the jitter by reducing the noise. More details about this architecture are reported in [11].

The architecture used to design the EVO flavors is shown in Fig. 4. It is based on a pass-band TIA with two amplification stages. The first stage consist of a buffered broadband amplifier in trans-impedance configuration followed by a cascode common source amplifier. The core of the first stage consists of cascode common source amplifier with ntype input transistor. A technique based on two branches is used to 


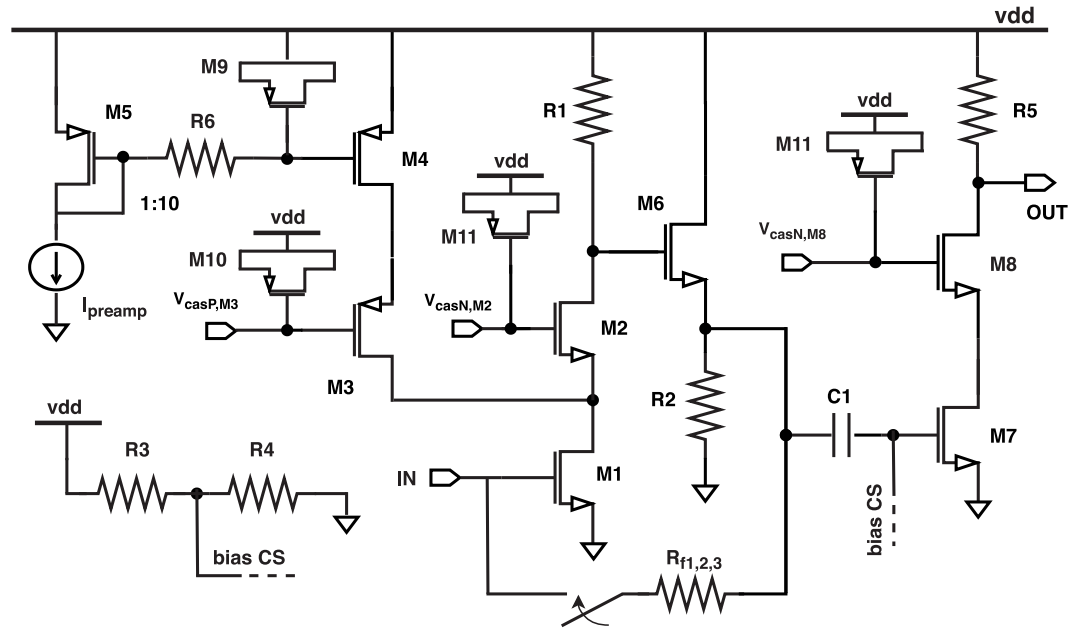

Fig. 4. Two stage TIA amplifier used in the EVO front-end.

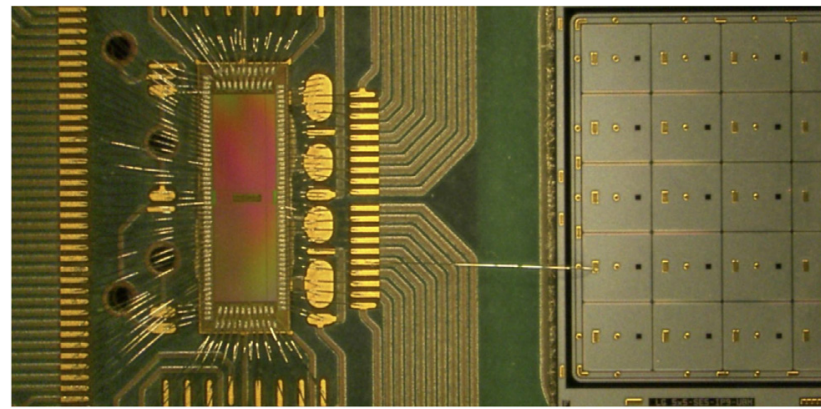

Fig. 5. Top view of FAST wire-bonded to the PCB pads and to a UFSD sensor.

Table 1

Main parameters defining the signal shaping of the EVO first-stage amplifier. $\mathrm{R}_{f}$ defines also to the low frequency gain.

\begin{tabular}{lll}
\hline $\mathrm{R}_{f}$ & Bandwidth & $\mathrm{R}_{\text {in }}$ \\
{$[\mathrm{k} \Omega]$} & {$[\mathrm{MHz}]$} & {$[\Omega]$} \\
\hline 5.3 & 665 & 90 \\
11.6 & 580 & 165 \\
31.6 & 230 & 490 \\
\hline
\end{tabular}

increase the open loop gain: the first branch (R1-M2) uses a small current $(\sim 80 \mu \mathrm{A})$ that allows keeping high the value of $\mathrm{R}_{1}$, which is important to increase the gain. The second branch (M4-M3), consisting of a cascode current mirror, provides a current up to $1.5 \mathrm{~mA}$ to $\mathrm{M} 1$, increasing its $g_{m}$ and then also the open-loop (OL) gain. The input transistor is designed to work in weak inversion mode to maximize $\mathrm{g}_{m}$. With a nominal current of $1 \mathrm{~mA}$, the OL gain of this stage is $35 \mathrm{~dB}$. The large bandwidth is obtained combining n-type cascodes (M2 and M8), minimizing the Miller effect. Moreover, passive loads (R1, R2 and R5) are adopted to avoid the parasitics introduced by active components.

The broadband is buffered by a wide-band n-type source follower. The TIA is obtained with the resistor $\mathrm{R}_{f}$ connected in feedback. Three values of $\mathrm{R}_{f}, 5.3 \mathrm{k} \Omega, 11.6 \mathrm{k} \Omega$ (nominal) and $31.6 \mathrm{k} \Omega$, can be connected by means of a dedicated configuration register. This allows exploring three different combinations of $\mathrm{BW}$, gain and $\mathrm{R}_{i n}$, as summarized in Table 1 . The architecture allows to tune the bandwidth between 230 and $665 \mathrm{MHz}$ allowing a peaking time $\mathrm{t}_{p, e l}$ between 0.49 and $1.2 \mathrm{~ns}$. The noise is reduced by using RC filters, R6-M9, and by a proper sizing of the input transistor. In nominal conditions, the noise at first stage output is $770 \mu \mathrm{V}$ RMS where the largest contribution comes from $\mathrm{R}_{f}$ itself.
The second stage is a cascoded common source with passive load. The input transistor works in weak inversion and its polarization is provided by a dedicated bias (R3 and R4). This stage is AC coupled to the first one, removing the low frequency variations present in the TIA output due to fabrication mismatch or leakage current in the sensors. The gain of the second stage is $13.7 \mathrm{~dB}$ and the bandwidth is large enough to maintain the timing properties of the first stage. With the nominal gain, the entire front-end exhibits a gain of $\sim 5.6 \times 10^{4} \mathrm{~V} / \mathrm{A}$, bandwidth of $460 \mathrm{MHz}$ and output noise of $2.7 \mathrm{mV}$ RMS. The EVO flavors are based on the architecture so far described but using different devices: standard and RF transistors have been used for EVO1 and EVO2, respectively.

\subsection{The FAST ASICs}

The flavors of the FAST family have been designed and produced in $110 \mathrm{~nm}$ CMOS technology. Each chip die fits an area of $1.6 \mathrm{~mm} \times 5 \mathrm{~mm}$ and contains 20 independent channels with an input pitch of $170 \mu \mathrm{m}$. The channel chain consists of a front-end (REG, EVO1 or EVO2), a leading-edge discriminator, a pulse width regulator (PWR) and a LVDS driver. The PWR is used to increase by a fixed quantity the Time-overThreshold length, to make FAST compatible with commercial TDC. The LVDS format is used for compatibility with commercial TDCs and FPGAs. More details about the chain can be found in [11]. Fig. 5 shows the FAST chip and a $5 \times 5$ UFSD array connected to the test board.

\section{FAST characterization}

\subsection{System-level simulations}

The FAST family is the result of system-level studies that include all terms, from the sensor and electronics side, affecting the time resolution. The study has also included the detailed emulation of the MIP charge deposition in the silicon sensors, including, for instance, the statistical effects in the sensor signal generation or the size of the sensor. An example of these studies is presented in Fig. 6, where the simulated $\mathrm{FAST}_{\mathrm{EVO} 2}$ front-end response to a $8 \mathrm{fC}$ delta-shaped input signal is plotted for different $\mathrm{C}_{\text {det }}$ values, ranging from 2 to $20 \mathrm{pF}$. The simulation shows that $\mathrm{C}_{d e t}$ affects significantly the signal amplitude because it impacts the impedance matching whereas the pulse duration does not change appreciably up to $10 \mathrm{pF}$. The study of these matching effects is carried out by connecting the FAST prototypes to different input capacitors while varying the rise time of the input signal. 

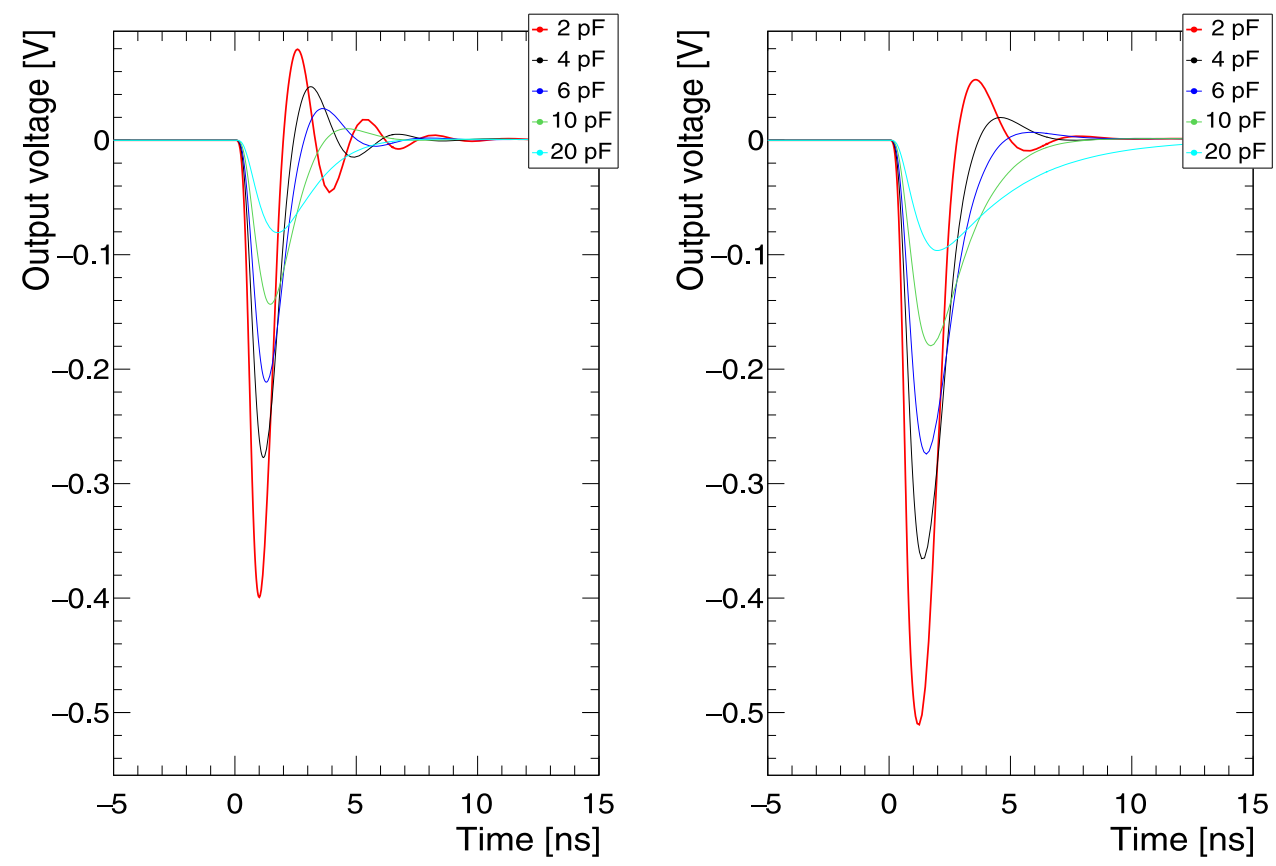

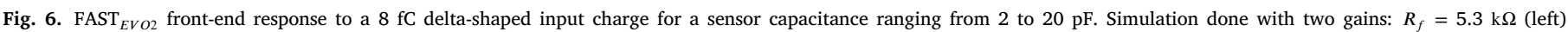
and $R_{f}=11.6 \mathrm{k} \Omega$ (right).

\subsection{Test setup and methodology}

A custom PCB test board, compatible with all FAST flavors, has been designed and fabricated. This PCB hosts the chip, the sensor, trimmers for the bias settings, and a 16-bits DAC for precise threshold voltage regulation (programmed by an external FPGA). The board allows detailed testing of the chip in stand-alone mode and in conjunction with the sensor (sensor-mode). In the stand-alone configuration, dedicated SMA connectors are used to inject current pulses to the FAST input through a $100 \mathrm{fF}$ series-connected capacitors $\mathrm{C}_{i n j}$. Input pulses in this setup are generated by a LeCroy 9210 pulser. The second test mode uses a pico-second laser to generate MIP-like current signals in a silicon sensor wire bonded to the FAST input pads. Depending on the tests setup, the FAST LVDS outputs are readout either using a differential probe connected to an oscilloscope (Tektronik DPO 7254, $2.5 \mathrm{GHz} \mathrm{BW}$ ) or using an FPGA board (Xilinx Kintex7-Ultrascale XCKU040) sampling the outputs at $1 \mathrm{GHz}$ (this is the same FPGA that controls the threshold setting). A fully-featured data acquisition system has been written in LabView. This DAQ system allows online data taking and analysis, including baseline searches and noise determination. The following section contains experimental results obtained with FAST $_{E V O 2}$ aiming at validate the system-level studies previously discussed.

\subsection{Experimental results of EVO2}

The $F A S T_{E V O 2}$ electronic noise, jitter, and time resolution have been measured in stand-alone mode and in sensor-mode. The measured power consumption is $\sim 14 \mathrm{~mW} / \mathrm{ch}$ where $2.6 \mathrm{~mW}$ is equally divided between front-end and discriminator, and the remaining part is devoted to the LVDS driver.

\subsubsection{Stand-alone measurements}

The jitter has been measured injecting input charges $Q_{i n}$ from $3 \mathrm{fC}$ to $50 \mathrm{fC}$. The input pulses are generated by sending to $\mathrm{C}_{i n j}$ voltage pulses with rising time $\mathrm{T}_{r, \text { in }}$ of $200 \mathrm{ps}$. In this configuration, the jitter is measured on the oscilloscope and it is defined as the RMS of the Time of Arrival (ToA), defined as the time delay between the trigger pulse and the $50 \%$ level of the LVDS output. The correct voltage pulse is obtained attenuating the trigger pulse by $16 \mathrm{~dB}$. In order to perform the

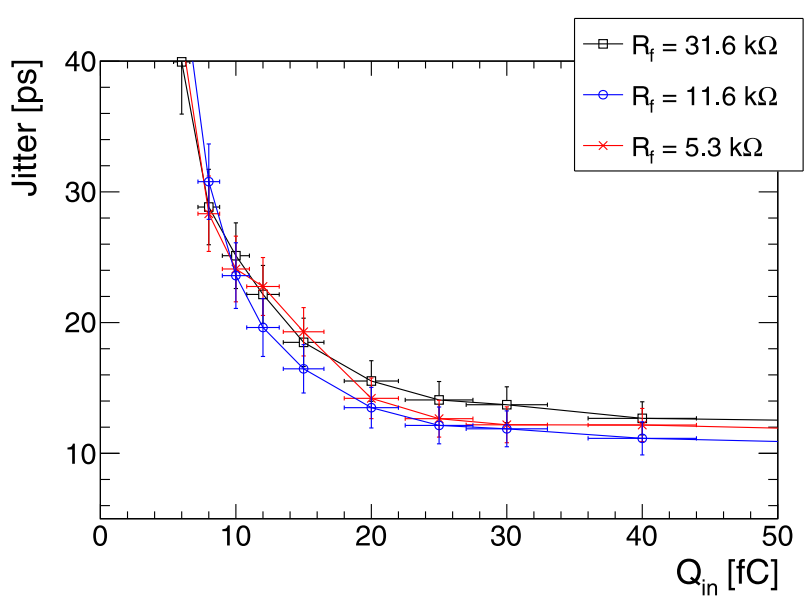

Fig. 7. Jitter for a range of charges injected with a pulser and the external capacitor $\mathrm{C}_{i n j}$. Measurement done with three gains.

measurement in a realistic configuration, a UFSD sensor biased beyond depletion having $4 \mathrm{pF}$ capacitance was connected to the input pad. Fig. 7 summarizes the results obtained with the three possible gain configuration, setting a threshold equal to $3 \mathrm{fC}$. The results are very similar in all cases, with a slightly better performances obtained with the medium gain (the nominal one), especially at large input charges. For $8 \mathrm{fC}$ input charge, the chosen working point used in the FAST design, the jitter is about 30 ps RMS and it saturates to 12 ps RMS at $50 \mathrm{fC}$. The jitter has also been measured as a function of the signal rise time through the regulation of the pulser $T_{r, i n}$, from 350 ps to $1 \mathrm{~ns}$, while keeping $\mathrm{Q}_{\text {in }}$ constant. Fig. 8 shows the results of this test for two input charges: 3.7 and $7.9 \mathrm{fC}$. In the first case, the jitter is measured to be between $20-36$ ps scanning the entire $T_{r, i n}$ range and between 20 and 30 ps with $7.9 \mathrm{fC}$.

The pulser setup has also been used to study the signal slope $\partial V / \partial t$ which defines the timing performance of the front-end. The reconstruction of the signal edge has been obtained by measuring with the oscilloscope the ToA as a function of the threshold voltage $\mathrm{V}_{T H}$. For 


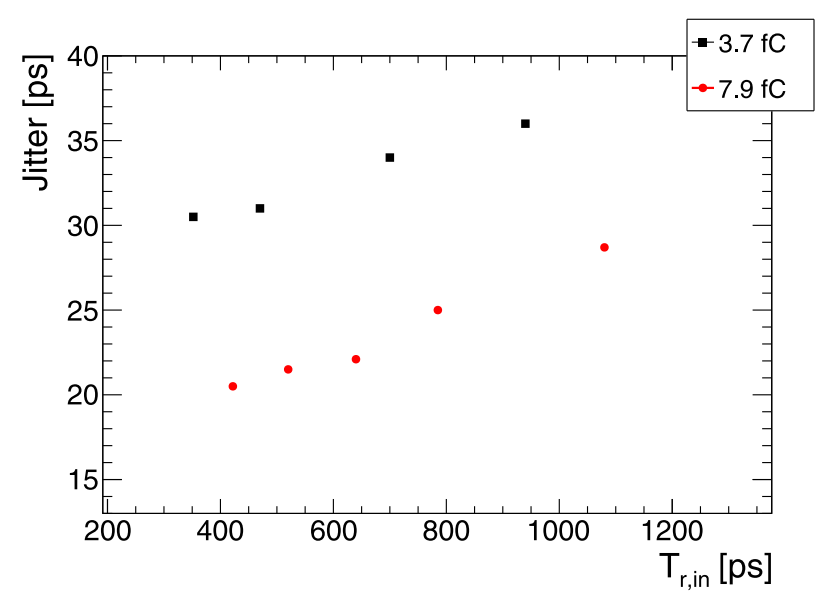

Fig. 8. Jitter measurement injecting 3.7 and $7.9 \mathrm{fC}$ with different values of $T_{r, i n}$. Measurement done without a sensor connected.

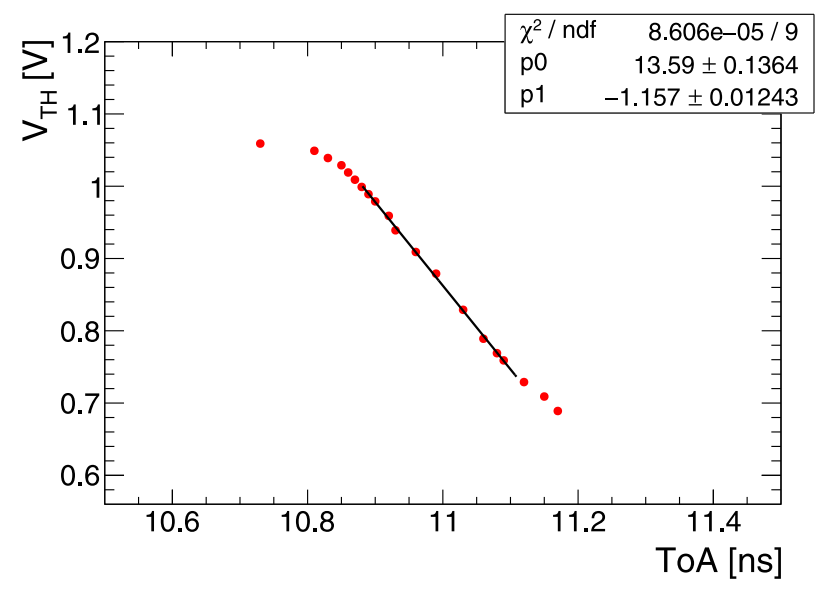

Fig. 9. Analog output reconstruction obtained with a threshold scan, $\mathrm{Q}_{\text {in }}$ of $24 \mathrm{fC}$ and input voltage pulse with $\mathrm{T}_{r, i n}$ of $500 \mathrm{ps}$.

this measurement, an input charge of $24 \mathrm{fC}$ has been generated with a voltage pulse with $T_{r, \text { in }} \sim 500$ ps. Fig. 9 shows the resulting signal reconstruction. The analog output exhibits a slope of $(-1.16 \pm 0.01) \mathrm{V} / \mathrm{ns}$, a peaking time $\mathrm{T}_{p}$ equal to $\sim 500 \mathrm{ps}$ and an amplitude of $370 \mathrm{mV}$. The baseline is measured to be at $(1063 \pm 2.9) \mathrm{mV}$ where $2.9 \mathrm{mV}$ is the RMS noise measured with a $4 \mathrm{pF}$ depleted sensor. The measured peaking time equals $T_{r, i n}$, demonstrating that the front-end does not introduce a significant distortion of the input pulse shape.

\subsubsection{Sensor-mode: $F A S T_{E V O 2}$ coupled to a UFSD}

The second setup consists of a FAST ${ }_{E V O 2}$ channel wire-bonded to a $55 \mu \mathrm{m}$ thick UFSD sensor, $1.3 \mathrm{~mm} \times 1.3 \mathrm{~mm}$ pad, with a $4 \mathrm{pF}$ capacitance (Fig. 5). The sensor bias voltage is provided by a HV power supply DT1419ET-CAEN. The full depletion of the sensor is expected to be reached at $41 \mathrm{~V}$. In this setup, the RMS noise has been measured by performing a threshold scan around the baseline of one channel of $\mathrm{FAST}_{\mathrm{EVO} 2}$ at increasing values of the bias voltage. The results, summarized in Fig. 10 show, as expected, that the noise is higher at low voltages due to the higher sensor capacitance. In particular, a RMS noise of $6.7 \mathrm{mV}$ is measured when the sensor is unbiased. From the known sensor properties, this situation corresponds to have a capacitance of $144 \mathrm{pF}$ connected to the input channel. The RMS noise decreases when the sensor bias increases and abruptly reach the minimum of $2.8 \mathrm{mV}$ when the sensor reach the full-depletion at $41 \mathrm{~V}$. This value is consistent with simulation results. For higher bias voltages, RMS noise is constant until reaching the breakdown voltage

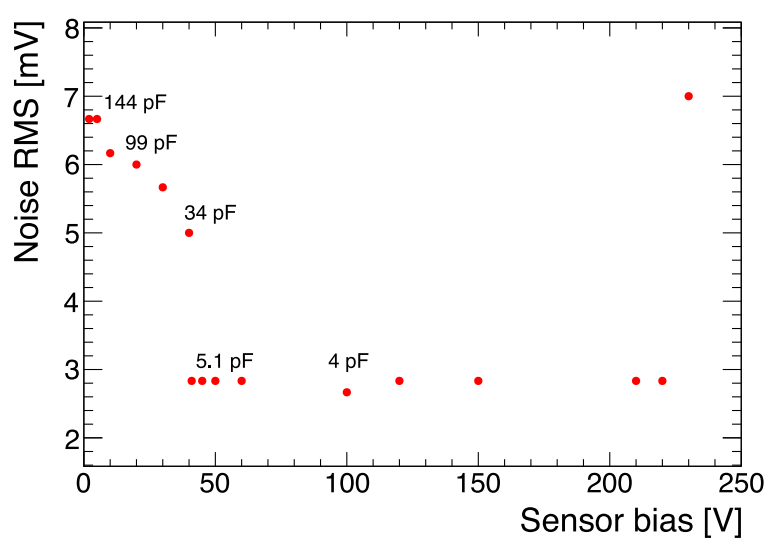

Fig. 10. RMS noise measurement on $\mathrm{FAST}_{E V O 2}$ coupled to a $4 \mathrm{pF}$ UFSD sensor for different sensor bias voltages.

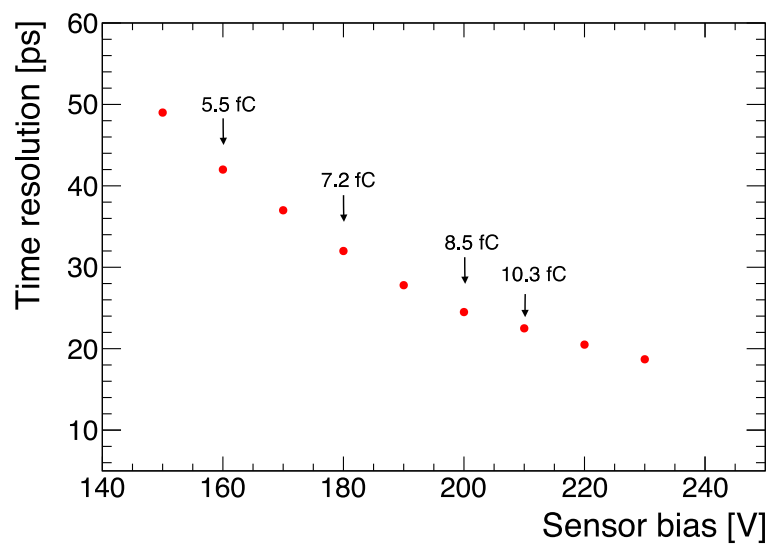

Fig. 11. Time resolution of FAST ${ }_{E V O 2}$ coupled to a $55 \mu \mathrm{m}$ UFSD, $4 \mathrm{pF}$ sensor. Charge regulated with the sensor bias regulation.

at $230 \mathrm{~V}$ where it abruptly increases due to increase of leakage current in the sensor. The RMS noise dependence on the input capacitance is weaker than $\sqrt{C_{d e t}}$.

The same sensor-mode setup has been used with a pico-second laser source $(\lambda=1020 \mathrm{~nm})$. The laser intensity has been tuned to provide to FAST the same charge generated by 1 MIP. The charge generated by the sensor has been regulated by controlling the sensor gain using the sensor bias voltage. With this setup, the time resolution has been measured as the ToA RMS for different sensor bias, up to $230 \mathrm{~V}$. The results, summarized in Fig. 11, show that the time resolution is a decreasing function of the input charge over the entire bias range. For an input charge of $5.5 \mathrm{fC}$ the time resolution is $42 \mathrm{ps}$ RMS while for the design point of $8.5 \mathrm{fC}$ the achieved resolution is $24.5 \mathrm{ps}$ RMS. The reported values assume the possible contributions to the jitter from the laser and/or oscilloscope to be negligible.

\section{Conclusions}

This paper reports results on the first prototypes of the FAST ASIC family. FAST (Fast Amplifiers for Silicon detectors Timing) has been designed and manufactured in CMOS $110 \mathrm{~nm}$ technology for high precision timing applications with UFSD. System-level simulations have been performed coupling the FAST architectures to the sensor, obtaining an expected jitter for 1 MIP signal in a $55 \mu \mathrm{m}$ thick UFSD (gain $=15$ ) of about $30 \mathrm{ps.} \mathrm{In} \mathrm{order} \mathrm{to} \mathrm{preserve} \mathrm{the} \mathrm{sensor} \mathrm{fast} \mathrm{signal}$ properties, a fast TIA amplifier architecture was used to design the two EVO flavors of the FAST family. FAST ${ }_{E V O 2}$ has been tested both in stand-alone mode and coupled with a UFSD sensor. The jitter, measured 
with a pulser setup, is about $30 \mathrm{ps}$ for the MPV charge expected from $1 \mathrm{MIP}$ in $55 \mu \mathrm{m}$ thick UFSD sensor with $4 \mathrm{pF}$ capacitance. As expected from simulations, a negligible dependency on the TIA gain has been observed. The analog signal reconstruction obtained with the typical gain shows a sharp signal-edge with a slope of $-1.16 \mathrm{~V} / \mathrm{ns}$ and a time to peak of $500 \mathrm{~ns}$ where the front-end contribution is negligible. The RMS noise, measured with a sensor connected to the input, is $2.8 \mathrm{mV}$, in agreement with simulations. Laser measurements on the sensor-chip system give a time resolution of 24.5 ps RMS for $8.5 \mathrm{fC}$ and $42 \mathrm{ps}$ RMS in the case of $5.5 \mathrm{fC}$. Results obtained on $\mathrm{FAST}_{E V O 2}$ with a $1.3 \mathrm{~mm} \times 1.3 \mathrm{~mm}$ UFSD fulfill the $30 \mathrm{ps}$ RMS target jitter and make this FAST flavor an interesting solution for pico-second time accuracy measurements applications.

\section{Declaration of competing interest}

The authors declare that they have no known competing financial interests or personal relationships that could have appeared to influence the work reported in this paper.

\section{Acknowledgments}

We kindly acknowledge the following funding agencies: European Union's Horizon 2020 Research and Innovation funding program Grant Agreement no. 669529, Italian Ministero degli Affari Esteri, INFN of
Turin Gruppo V and the MIUR, Physics Dep. of Torino (ex L. 232/2016, art. 1, cc. 189 314, 337), Physics Dep. of Torino.

\section{References}

[1] G. Soyez, Pileup mitigation at the LHC, 2019, arXiv:1801.09721v2.

[2] ATLAS Collaboration, Technical Design Report for the ATLAS Inner Tracker Pixel Detector, CERN-LHCC-2017-021 ATLAS-TDR-030.

[3] CMS Collaboration, A MIP timing Detector for the CMS Phase-2 upgrade, CMS-TDR-020, 2019.

[4] H.F.-W. Sadrozinski, et al., Ultra-fast silicon detectors (UFSD), Nucl. Instrum. Methods A 831 (2016).

[5] H.F.-W. Sadrozinski, Abraham Seiden, Nicolò Cartiglia, 4D tracking with ultra-fast silicon detectors, Rep. Progr. Phys. 81 (2018) 026101.

[6] F. Cenna, et al., TOFFEE: a full custom amplifier-comparator chip for timing applications with silicon detectors, JINST 12 (2017) C03031.

[7] F. Anghinolfi, et al., NINO: An ultrafast Low-Power Front-End Amplifier discriminator for the time-of-flight detector in the ALICE experiment, IEEE Trans. Nucl. Sci. 51 (5) (2004).

[8] C. De La Taille, et al., ALTIROC0, a 20 pico-second time resolution ASIC for the ATLAS High Granularity Timing Detector (HGTD), PoS TWEPP-17 (2018) 006.

[9] A. Rivetti, Front-End Electronics for Radiation Sensors, CRC Press, 2015.

[10] H. Spieler, Fast timing methods for semiconductor detectors, IEEE Trans. Nucl. Sci. 29 (3) (1982).

[11] E.J. Olave, et al., A multichannel front-end readout ASIC for picosecond time resolution systems based on thin UFSD, JINST (2019). 